\title{
A novel mutation of the FAT2 gene in spinal meningioma
}

\author{
GENSHU TATE $^{1,2}$, KOJI KISHIMOTO ${ }^{1}$ and TOSHIYUKI MITSUYA ${ }^{1,3}$ \\ ${ }^{1}$ Department of Pathology, Showa University Fujigaoka Hospital, Yokohama, Kanagawa 227-8501; \\ ${ }^{2}$ Department of Pathology, Showa University, Tokyo 142-8555; ${ }^{3}$ Department of Pathology, \\ Kokusai-Shinzen Hospital, Yokohama, Kanagawa 245-0006, Japan
}

Received March 1, 2015; Accepted March 15, 2016

DOI: $10.3892 / \mathrm{ol} .2016 .5063$

\begin{abstract}
Meningiomas may be classified as neurofibromin 2 (NF2)-associated and non-NF2 meningiomas depending on the presence or absence of molecular alterations in the $N F 2$ gene. One of the characteristic histological features of meningiomas is the whorl formation of neoplastic arachnoid cells. NF2 is a human homolog of the Drosophila gene, Merlin (Mer). In humans, NF2 is the gene responsible for the disease neurofibromatosis type II, which results in the development of brain tumors, including acoustic neurinoma and meningioma. The present study aimed to investigate the molecular pathogenesis of spinal meningioma. It was hypothesized that the whorl formation of meningiomas may occur as a result of a disturbance in the planar cell polarity (PCP) of arachnoid cells, thus, genes understood to govern PCP signaling were analyzed for alterations. Whole exome sequencing followed by Sanger sequencing validation was performed for the analysis of spinal meningioma tissue obtained from a 42-year-old Japanese female. The sequencing identified a nonsynonymous mutation of c.3597G $>$ C, resulting in p.Q1199H, in the FAT atypical cadherin 2 (FAT2) gene. FAT2 is homologous to the Drosophila Fat $(F t)$ gene, which belongs to the cadherin superfamily. Drosophila Fat is involved in PCP, tumor suppression and Hippo (Hpo) signaling, which is associated with Mer. Taken together, the results of the present study concluded that human FAT2 may function as a key molecule that governs not only PCP, but also NF2-Hpo signaling in arachnoid cells; thus, a mutation in this gene may result in spinal meningioma.
\end{abstract}

\section{Introduction}

Meningiomas arise from meningeal arachnoid cells. A key histological characteristic associated with these lesions is the concentric arrangement of meningioma cells around

Correspondence to: Dr Genshu Tate, Department of Pathology, Showa University Fujigaoka Hospital, 1-30 Fujigaoka, Aobaku-ku, Yokohama, Kanagawa 227-8501, Japan

E-mail: gentate@yahoo.co.jp

Key words: FAT atypical cadherin 2 gene, spinal meningioma, mutation calcified tissue, known as psammoma bodies, to form a whorl appearance. Microscopically, the World Health Organization (WHO) classification scheme recognizes 15 variations of meningioma, which can be classified into benign (grade I), atypical (grade II) and malignant (grade III). Approximately $90 \%$ of all meningiomas fall into the grade-I category, which includes nine histological subtypes: Meningothelial, fibrous (fibroblastic), transitional (mixed), psammomatous, angiomatous, microcystic, secretory, lymphoplasmacyte-rich and metaplastic. Based on molecular pathogenesis, meningiomas are classified as neurofibromin 2 [NF2; Drosophila Merlin (Mer)] mutant meningiomas (NF2-associated meningiomas) and non-NF2 meningiomas, with the loss of NF2 observed in $40-60 \%$ of sporadic meningioma cases $(1,2)$. Hedgehog (Hh) signaling molecules are considered to be involved in the pathogenesis of meningioma due to the detection of suppressor of fused (SUFU), a component of the Hh signaling pathway, in familial multiple meningioma (3). However, the frequency of mutations in SUFU is extremely low, with no SUFU mutations detected in a study of 162 individuals with sporadic meningioma (3). Previously, next-generation sequencing analysis of non-NF2 meningiomas identified novel, recurrent mutations in the following genes: TNF receptor-associated factor 7, Krüppel-like factor 4, v-akt murine thymoma viral oncogene homolog 1 and smoothened, frizzled class receptor $(1,2)$. In addition, it was demonstrated that NF2 meningiomas are associated with the Hippo (Hpo) pathway, which regulates tissue growth, proliferation and ultimately controls organ size (4-6).

The present study hypothesized that the whorl appearance of meningioma may occur as a result of a disturbance in planar cell polarity (PCP), and the molecular pathogenesis of the whorl appearance may involve genes that control PCP. In Drosophila, the PCP signaling molecules have been well characterized, and include Frizzled [human homologs, frizzled class receptor (FZD)3 and FZD6], Dishevelled [human homologs, dishevelled segment polarity protein (DVL)1, DVL2 and DVL3], Flamingo [human homologs, cadherin, EGF LAG seven-pass G-type receptor (CELSR)1, CELSR2 and CELSR3], Diego (human homolog, ankyrin repeat domain 6), Van Gogh [human homologs, VANGL planar cell polarity protein (VANGL)1 and VANGL2] and Prickle [human homologs, prickle planar cell polarity protein (PRICKLE)1 and PRICKLE2] (7). Fat (Ft)-Hpo signaling is also involved in PCP (7). The Ft-Hpo signaling molecules in Drosophila are as follows: Ft [human homologs, FAT atypical cadherin (FAT)1, 
A

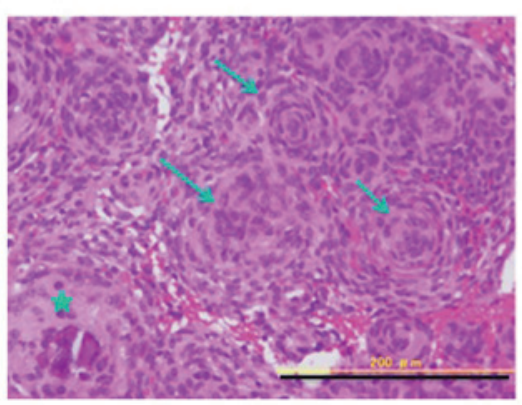

B

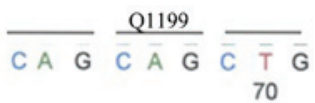

C $\overline{\overline{C A G}} \frac{\text { Q1199H }}{C A G} \overline{C T G}$
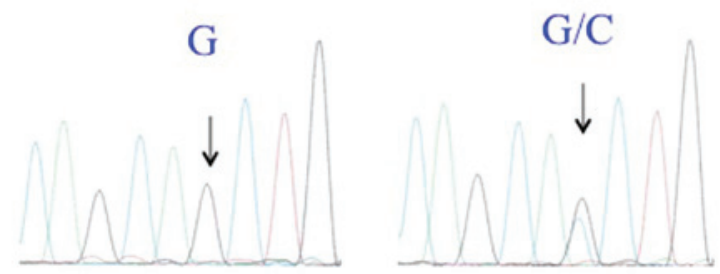

D

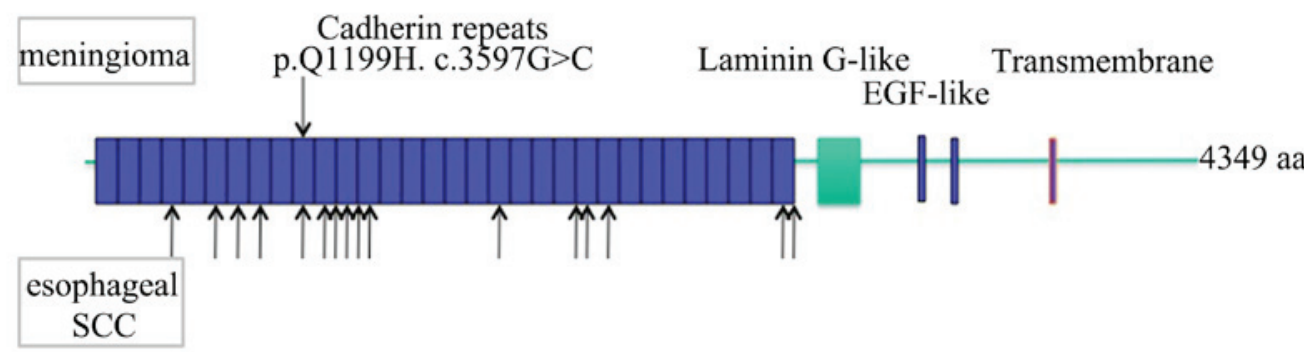

Figure 1. (A) Histopathological features of a grade I spinal meningioma (classified according to the World Health Organization classification system) exhibiting whorl formations (arrows) and a psammoma body (star) (hematoxylin and eosin staining; scale bar, $200 \mu \mathrm{m}$ ). Sanger sequencing confirmed (B) wild-type c.3597G in germline analysis of the FAT2 gene, whereas (C) a c.3597G >C mutation was observed in the FAT2 gene of the spinal meningioma, resulting in p.Q1199H. (D) Schematic comparisons of FAT2 mutations between esophageal SCC and spinal meningioma. The arrows indicate the locations of mutations. The upward arrows in the cadherin repeats represent mutations of the FAT2 gene previously identified in esophageal SCC (15), whilst the downward arrow represents the mutation identified in the present study. EGF, epidermal growth factor; FAT2, FAT atypical cadherin 2; SCC, squamous cell carcinoma.

FAT2, FAT3 and FAT4], Dachsous [human homologs, dachsous cadherin-related (DCHS)1 and DCHS2], Four-jointed (human homolog, four jointed box 1), Dachs, Expanded [human homologs, FERM domain containing (FRMD)1 and FRMD2], Mer (human homolog, NF2), Kibra ortholog [human homologs, WW and $\mathrm{C} 2$ domain containing (WWC)1 and WWC2], Salvador (human homolog, salvador family WW domain containing protein 1), Hpo [serine/threonine kinase (STK)4 and STK3], Warts [human homologs, large tumor suppressor kinase (LATS)1 and LATS2], Mats [human homologs, MOB kinase activator (MOB)1 A and MOB1B], Yorkie (human homologs, yes-associated protein 1 and tafazzin) and Scalloped [human homologs, TEA domain transcription factor (TEAD)1, TEAD2, TEAD3 and TEAD4] $(8,9)$. The current study performed whole exome sequencing (WES) analysis to identify mutated genes in spinal meningioma by screening the human homologs of Drosophila PCP genes, in addition to Ft-Hpo signaling genes. A nonsynonymous mutation was identified in the FAT2 gene in a non-NF2 meningioma, thus indicating a possible association between the FAT2 gene and the molecular pathogenesis of meningioma.

\section{Patient and methods}

A 42-year-old female patient was admitted to Showa University Fujigaoka Hospital (Yokohama, Japan) in November 2013 after experiencing loss of consciousness and falling down twice within 2 weeks. Approximately 1 month later, marked muscle weakness was detected in the left leg. Magnetic resonance imaging of the thorax, which was performed with a Signa HDxt 1.5T (GE Healthcare Japan Corporation, Tokyo, Japan), revealed a tumor in the extramedullary spinal cord and intradural space at the $\mathrm{C} 7$ to Th1 level. Surgery was performed to fully remove the tumor, which was $\sim 1 \mathrm{~cm}$ in diameter, and histopathological analysis confirmed a diagnosis of spinal meningioma, which exhibited numerous psammoma bodies and a whorl formation of the neoplastic arachnoid cells (Fig. 1A). For the analysis, the tissue was formalin-fixed, and formalinfixed paraffin-embedded sections of $3 \mu \mathrm{m}$ in thickness were prepared with a CRM-440 microtome (Sakura Seiki Co., Ltd., Tokyo, Japan), and subsequently stained with hematoxylin and eosin (Merck Japan, Tokyo, Japan) as previously described (10). Olympus microscope BX60 and DP73 digital camera (Olympus Corporation, Tokyo, Japan) with WinROOF2013 software (Mitani Valve Co., Ltd., Tokyo, Japan) were used for visual examination. According to the WHO classification system (11), the tumor was a grade I meningothelial meningioma.

Genomic DNA was extracted from the meningioma tissue by phenol-chloroform extraction and ethanol precipitation methods (12), and was subsequently used for WES and Sanger sequencing. Peripheral blood mononuclear cells were used as a DNA source for germline sequence analysis. Next-generation WES using exome capture via in-solution hybridization followed by massive parallel sequencing were conducted as previously described (13). Briefly, $\sim 3 \mu \mathrm{g}$ of genomic DNA was sheared to a mean fragment size of $300 \mathrm{bp}$, and the fragments were used for Illumina Paired-End DNA library preparation and enrichment of target sequences (Agilent Technologies, Inc., Santa Clara, CA, USA). Exon capture was then performed using the SureSelect Human All Exon v4 kit (Agilent Technologies, Inc.). The mean size of the sequence library was $450 \mathrm{bp}$, and the enriched DNA fragments were sequenced with 100-bp paired-end reads in 
the HiSeq 2000 sequencing system (Illumina, Inc., San Diego, CA, USA). The sequencing reads were aligned to the reference human genome (1000 Genomes; http://www.1000genomes.org/) using the Genome Analysis Toolkit (https://software.broadinstitute.org/gatk/) and Burrows-Wheeler Aligner software (http:// bio-bwa.sourceforge.net/). Single-nucleotide substitutions and small insertions-deletions (indels) were annotated against the RefSeq database (http://www.ncbi.nlm.nih.gov/refseq/) and The Single Nucleotide Polymorphism database 137 (http:// www.ncbi.nlm.nih.gov/SNP/) using the ANNOVAR tool (http:// annovar.openbioinformatics.org/en/latest/).

In order to validate any mutations, Sanger sequencing was also performed. The primers used for FAT2 amplification were as follows: Forward, 5'-TCTTGAAGTTGCCTC AGTAAAGT-3' and reverse, 5'-CTAACATGGCTCCACAAA TCACC-3'. Polymerase chain reaction (PCR) was conducted with 30 cycles of denaturation $\left(98^{\circ} \mathrm{C}\right.$ for $\left.1 \mathrm{~min}\right)$, annealing $\left(65^{\circ} \mathrm{C}\right.$ for $2 \mathrm{~min}$ ) and extension $\left(72^{\circ} \mathrm{C}\right.$ for $\left.3 \mathrm{~min}\right)$. PCR was performed in a Quick Thermo Personal thermal cycler (Nippon Genetics Co., Ltd., Tokyo, Japan), using deoxynucleotides (Takara Bio, Inc., Otsu, Japan) and Tris-borate-ethylenediaminetetraacetic acid (Takara Bio, Inc.) as a buffer. Amplified DNA fragments were recovered from a low melting temperature agarose gel (2\% Agarose L; Wako Pure Chemical Industries, Ltd,. Osaka, Japan) and subjected to direct sequencing analysis using an automated ABI PRISM ${ }^{\circledR} 377$ DNA Sequencer (Applied Biosystems; Thermo Fisher Scientific, Inc., Waltham, MA, USA), as previously described (13).

Written informed consent was obtained from the patient. The present study complied with the Declaration of Helsinki revised in 2008 (14), and was conducted according to the ethical guidelines presented annually by the Committee for Medical Experiment at Showa University.

\section{Results}

WES analysis identified no alterations of the NF2 gene (22q12.2). Therefore, human genes homologous to those involved in PCP and Ft-Hpo signaling in Drosophila were analyzed (9). Subsequent to screening of the WES data and validation by Sanger sequencing, a nonsynonymous mutation in exon 4 of the FAT2 gene, c. $3597 \mathrm{G}>\mathrm{C}$, that resulted in p.Q1199H, was identified in spinal meningioma tissue, which was not detected in the germline sequence, indicating the heterozygous mutation of the FAT2 gene in spinal meningioma (Fig. 1B and C). The spinal meningioma mutation of the FAT2 gene was located in the 10th of 32 cadherin repeats, although the same mutation was not identified to be common to both esophageal squamous cell carcinoma (ESCC) and the present case, as indicated by the arrows in Fig. 1D. No other mutations were detected among the PCP or Ft-Hpo signaling genes.

\section{Discussion}

Psammoma bodies observed in meningioma tissue exhibit similar histological characteristics to those observed in well-differentiated ESCC whose tumor cells form a whorl-like arrangement, known as keratin pearls. These similarities in cell arrangement in meningioma and esophageal SCC tissue indicate that the horizontal cell polarity, which is controlled by cell surface adhesion molecules (including cadherin family members), may be disturbed in these tumors, whereby the tumor cells proliferate towards the center of the tumor nest (11). Lin et al (15) identified 34 mutations of human FAT genes, FAT1, FAT2 and FAT3, in 139 cases of esophageal SCC. FAT2 gene mutations were reported in 12 of these cases $(8$ cases of missense and 4 cases of stop-gain, splicing site, frameshift or indel), although no information regarding the different histological types of esophageal SCC are currently available (15).

In vitro and in vivo experiments have demonstrated the involvement of FAT2 in the molecular pathogenesis of SCC. Matsui et al (12) reported that human FAT2 is localized at immature adherens junctions in epidermal keratinocytes, and the knockdown of human FAT2 by siRNA inhibited the migration of the cultured HSC-1 human SCC cell line. Furthermore, Lin et al (15) demonstrated that the depletion of FAT2 with small hairpin RNA promoted esophageal SCC growth in vivo.

FAT2 is a member of the cadherin superfamily and is homologous to Drosophila $\mathrm{Ft}$, which functions as a positive regulator of PCP in the Drosophila wing $(8,9)$. The human FAT2 gene (5q33.1) encodes a large, type I transmembrane protein belonging to the cadherin superfamily, which consists of 4,349 amino acids with an extracellular domain (amino acids 19-4,048), transmembrane region (amino acids 4,049-4,069) and cytoplasmic domain (amino acids 4,070-4,349). The extracellular domain consists of 32 cadherin repeats, two epidermal growth factor-like domains and one laminin G-like domain (9). The FAT2 mutation identified in the present study was located in the cadherin repeats. How the signaling of FAT2 with p.1199H differs from that of the wild-type remains to be elucidated.

The present case of meningioma was classified as WHO grade I, thus, indicating that the FAT2 mutation may be an initial or early genetic alteration, as the number of mutated genes in grade I meningioma is considered to be limited compared with that in high-grade meningioma.

Immunohistochemistry is regarded as a simple method to detect FAT2 expression in meningioma. At present, however, no specific antibodies against human FAT2 are commercially available. Thus, once an appropriate antibody for use with formalin-fixed, paraffin-embedded sections has been developed, the performance of routine medical examination to detect how FAT2 expression differs among varying grades of meningioma is anticipated to improve. In addition, in vitro analyses may aid investigation into how FAT2 with p.1199H differs in its PCP signaling compared with the wild-type protein in arachnoid cells.

In conclusion, the present study identified the presence of a novel FAT2 somatic mutation in a non-NF2 spinal meningioma, indicating that the Hpo signaling pathway is important in NF-2-associated and non-NF2 meningioma. Additionally, it was hypothesized that a mutation of FAT2 may be involved in the molecular pathogenesis of non-NF2 meningioma, potentially providing a molecular target for novel therapeutic drugs for the treatment of patients with meningioma.

\section{References}

1. Brastianos PK, Horowitz PM, Santagata S, Jones RT, McKenna A, Getz G, Ligon KL, Palescandolo E, Van Hummelen P, Ducar MD, et al: Genomic sequencing of meningiomas identifies oncogenic SMO and AKT1 mutations. Nat Genet 45: 285-289, 2013. 
2. Clark VE, Erson-Omay EZ, Serin A, Yin J, Cotney J, Ozduman K, Avşar T, Li J, Murray PB, Henegariu O, et al: Genomic analysis of non-NF2 meningiomas reveals mutations in TRAF7, KLF4, AKT1 and SMO. Science 339: 1077-1080, 2013.

3. Aavikko M, Li SP, Saarinen S, Alhopuro P, Kaasinen E, Morgunova E, Li Y, Vesanen K, Smith MJ, Evans DG, et al: Loss of SUFU function in familial multiple meningioma. Am J Hum Genet 91: 520-526, 2012.

4. Zhang N, Bai H, David KK, Dong J,Zheng Y, Cai J, Giovannini M, Liu P, Anders RA and Pan D: The Merlin/NF2 tumor suppressor functions through the YAP oncoprotein to regulate tissue homeostasis in mammals. Dev Cell 19: 27-38, 2010.

5. Liu AM, Wong KF, Jiang X, Qiao Y and Luk JM: Regulators of mammalian Hippo pathway in cancer. Biochim Biophys Acta 1826: 357-364, 2012.

6. Dong J, Feldmann G, Huang J, Wu S, Zhang N, Comerford SA, Gayyed MF, Anders RA, Maitra A and Pan D: Elucidation of a universal size-control mechanism in Drosophila and mammals. Cell 130: 1120-1133, 2007.

7. Viktorinová I, Pismen LM, Aigouy B and Dahmann C: Modelling planar polarity of epithelia: The role of signal relay in collective cell polarization. J R Soc Interface 8: 1059-1063, 2011.

8. Viktorinová I, König T, Schlichting K and Dahmann C: The cadherin Fat 2 is required for planar cell polarity in the Drosophila ovary. Development 136: 4123-4132, 2009.
9. Katoh M: Function and cancer genomics of FAT family genes (review). Int J Oncol 41: 1913-1918, 2012.

10. Lyle HM: An improved tissue technique with hematoxylin-eosin stain. Am J Med Technol 13: 178-181, 1947.

11. Louis DN, Ohgaki H, Wiestler OD, Cavenee WK, Burger PC, Jouvet A, Scheithauser BW and Kleihues P: The 2007 WHO classification of tumors of the central nervous system. Acta Neuropathol 114: 97-109, 2007.

12. Matsui S, Utani A, Takahashi K, Mukoyama Y, Miyachi Y and Matsuyoshi N: Knockdown of Fat2 by siRNA inhibits the migration of human squamous carcinoma cells. J Dermatol Sci 51: 207-210, 2008.

13. Tate G, Tajiri T, Kishimoto K and Mitsuya T: A novel mutation of the axonemal dynein heavy chain gene 5 (DNAH5) in a Japanese neonate with asplenia syndrome. Med Mol Morphol 48: 116-122, 2015.

14. Williams JR: The Declaration of Helsinki and public health. Bull World Health Organ 86: 650-652, 2008.

15. Lin DC, Hao JJ, Nagata Y, Xu L, Shang L, Meng X, Sato Y, Okuno Y, Varela AM, Ding LW, et al: Genomic and molecular characterization of esophageal squamous cell carcinoma. Nat Genet 46: 467-473, 2014. 